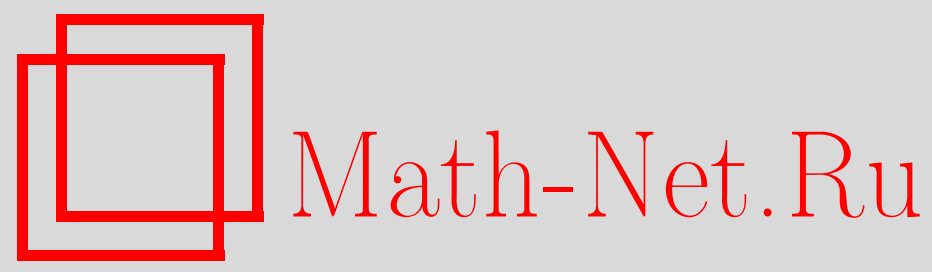

А. Ю. Ухалов, Почти периодические решения систем дифференциальных уравнений с быстрым и медленным временем в случае вырождения, Матем. заметки, 1998, том 63, выпуск 3, 451-456

DOI: https://doi.org/10.4213/mzm1302

Использование Общероссийского математического портала Math-Net.Ru подразумевает, что вы прочитали и согласны с пользовательским соглашением http://www . mathnet.ru/rus/agreement

Параметры загрузки:

IP : 54.89 .56 .158

26 апреля 2023 г., 13:06:24

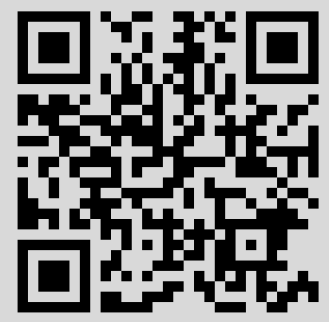




\section{ПОЧТИ ПЕРИОДИЧЕСКИЕ РЕШЕНИЯ СИСТЕМ ДИФФЕРЕНЦИАЛЬНЫХ УРАВНЕНИЙ С БЫСТРЫМ И МЕДЛЕННЫМ ВРЕМЕНЕМ В СЛУЧАЕ ВЫРОЖДЕНИЯ}

\section{А. Ю. Ухалов}

Рассматривается система обыкновенных дифференциалных уравнений первого порядка. Правые части системы пропорциональны малому параметру, почти периодически зависят от быстрого времени и периодически от медленного времени. Этой системе сопоставляется усредненная по быстрому времени система. Предполагается, что усредненная система имеет негрубое периодическое решение. Доказывается теорема существования и устойчивости почти периодических решений исходной системы.

Библиография: 6 названий.

1. Рассмотрим систему обыкновенных дифференциальных уравнений

$$
\frac{d x}{d t}=\varepsilon X(t, \tau, x, \varepsilon), \quad \tau=\varepsilon t
$$

где $x \in \mathbb{R}^{n}, \varepsilon \in\left[0, \varepsilon^{*}\right)$ - малый параметр, $X(t, \tau, x, \varepsilon)$ - вектор-функция, почти периодическая по $t$ равномерно относительно остальных аргументов, периодическая по $\tau$ с периодом $\omega$ и достаточно гладкая по $\tau, x$ и $\varepsilon$.

Будем обозначать через $B\left(\mathbb{R}^{n}\right)$ банахово пространство почти периодических функций со значениями в $\mathbb{R}^{n}$, через $|x|$ - евклидову норму в $\mathbb{R}^{n}$ и через $\|x(t)\|$ - норму в $B\left(\mathbb{R}^{n}\right)$. Шар $\|x-a\| \leqslant r$ пространства $B\left(\mathbb{R}^{n}\right)$ с центром в $a$ радиуса $r$ обозначим $U(a, r)$. В дальнейшем вместо “почти периодическая функция" будем писать “п.п.-функция".

Системе (1) сопоставим усредненную систему

$$
\frac{d y}{d \tau}=Y_{1}(\tau, y), \quad \tau=\varepsilon t
$$

где

$$
Y_{1}(\tau, y)=\lim _{T \rightarrow \infty} \frac{1}{T} \int_{0}^{T} X(t, \tau, y, 0) d t .
$$

Пусть система (2) имеет $\omega$-периодическое решение $y_{0}(\tau)$. Известно (см. [1]), что если линейная периодическая система

$$
\frac{d y}{d \tau}=A(\tau) y, \quad \text { где } \quad A(\tau)=Y_{1 y}^{\prime}\left(\tau, y_{0}(\tau)\right),
$$


не имеет характеристических показателей с нулевой вещественной частью, то система (1) при достаточно малых $\varepsilon$ имеет единственное в шаре $U\left(y_{0}(\varepsilon t), b(\varepsilon)\right)$ п.п.-решение $x(t, \varepsilon)$, причем

$$
\lim _{\varepsilon \rightarrow 0}\left\|x(t, \varepsilon)-y_{0}(\varepsilon t)\right\|=0 .
$$

Решение $x(t, \varepsilon)$ экспоненциально устойчиво, если все характеристические показатели системы (3) имеют отрицательные вещественные части, и неустойчиво в противном случаe.

В настоящей работе исследуется вопрос о сушествовании п.п.-решения, близкого к $y_{0}(\tau)$ при малых $\varepsilon$, в случае, когда система (3) имеет характеристическиепоказатели с нулевой вещественной частью. В частном случае, когда система (3) имеет один простой мультипликатор, равный по модулю 1, эта задача изучалась в работе [2]. В [2] дополнительно предполагалось, что $y_{0}(\tau) \equiv 0$ и что система $(1)$ имеет нулевое решение, т.е. рассматривалась задача о бифуркации малых п.п.-решений из состояния равновесия.

2. Предположим, что система (1) с помощью замены

$$
x=y+\sum_{k=1}^{m} \varepsilon^{k} u_{k}(t, \tau, y)
$$

где $u_{k}(t, \tau, y)$ - почти периодические по $t$ и $\omega$-периодические по $\tau$ функции, может быть преобразована к виду

$$
\frac{d y}{d \tau}=\sum_{k=1}^{m} \varepsilon^{k-1} Y_{k}(\tau, y)+\varepsilon^{m} F\left(\frac{\tau}{\varepsilon}, \tau, y, \varepsilon\right), \quad \tau=\varepsilon t .
$$

Здесь $Y_{k}(\tau, y)-\omega$-периодические по $\tau$ вектор-функции, достаточно гладкие по $\tau$ и $y$, $F(s, \tau, y, \varepsilon)$ - вектор-функция, почти периодическая по $s$ равномерно относительно остальных переменных, $\omega$-периодическая по $\tau$ и достаточно гладкая по $y$ и $\varepsilon$.

Запишем систему (1) в виде

$$
\frac{d x}{d t}=\sum_{k=1}^{m} \varepsilon^{k} X_{k}(t, \tau, x)+\varepsilon^{m+1} G(t, \tau, x, \varepsilon) .
$$

Сделав в (6) замену (4) и приравняв слагаемые при одинаковьх степенях $\varepsilon$, получим для определения $Y_{k}(\tau, y)$ и $u_{k}(t, \tau, y)$ следующую систему уравнений:

$$
\frac{\partial u_{k}}{\partial t}=\Omega_{k}(t, \tau, y)-Y_{k}(\tau, y), \quad k=1, \ldots, m .
$$

Здесь $\Omega_{1}(t, \tau, y)=X_{1}(t, \tau, y)$, а функции $\Omega_{i}(t, \tau, y)(i=2, \ldots, m)$ выражаются через правые части системы $(6)$ и функции $u_{1}(t, \tau, y), \ldots, u_{i-1}(t, \tau, y), Y_{1}(\tau, y), \ldots, Y_{i-1}(\tau, y)$. Например,

$$
\Omega_{2}(t, \tau, y)=X_{2}(t, \tau, y)+X_{1_{x}^{\prime}}^{\prime}(t, \tau, y) u_{1}(t, \tau, y)-u_{1_{\tau}^{\prime}}^{\prime}(t, \tau, y)-u_{1}^{\prime}(t, \tau, y) Y_{1}(\tau, y) .
$$

В уравнениях (7) $\tau$ и $y$ являются параметрами, а не функциями $t$. Коэффициенты замены $u_{i}(t, \tau, y)(i=1, \ldots, m)$ могут быть найдены последовательно, начиная с $u_{1}(t, \tau, y)$, непосредственным интегрированием. Необходимым условием почти периодичности 
$u_{i}(t, \tau, y)$ по $t$ является равенство нулю среднего значения по $t$ правой части $i$-го уравнения (7). Из этого условия определяется $Y_{i}(\tau, y)$ : нужно положить

$$
Y_{i}(\tau, y)=\lim _{T \rightarrow \infty} \frac{1}{T} \int_{0}^{T} \Omega_{i}(t, \tau, y) d t .
$$

После этого находится $u_{i}(t, \tau, y)$ как решение $i$-го уравнения $(7)$ с нулевьм средним значением по $t$.

ЗАмЕчАниЕ. Условие (8) является необходимым, но не достаточным, для того чтобы интеграл от правой части (7) являлся п.п.-функцией по $t$ (см. [3, гл. $1, \S 2])$. Мы будем предполагать, что функции $\Omega_{i}(t, \tau, y)$ в $(7)$ такие, что $u_{i}(t, \tau, y)$ - п.п.-функции по переменной $t$. (Так будет, в частности, если $X_{i}(t, \tau, y)(i=1, \ldots, m)$ в (6) являются тригонометрическими многочленами по $t$.)

3. Пусть система $m$-го приближения

$$
\frac{d y}{d \tau}=\sum_{k=1}^{m} \varepsilon^{k-1} Y_{k}(\tau, y), \quad \tau=\varepsilon t,
$$

при достаточно малых $\varepsilon$ имеет $\omega$-периодическое решение $y_{0}(\tau, \varepsilon)$, причем $y_{0}(\tau, 0)=y_{0}(\tau)$. (Здесь $y_{0}(\tau)-\omega$-периодическое решение системы первого приближения (2).) Рассмотрим линеаризованную на $y_{0}(\tau, \varepsilon)$ систему $(9)$

$$
\frac{d y}{d \tau}=A(\tau, \varepsilon) y, \quad \text { где } A(\tau, \varepsilon)=\sum_{k=1}^{m} \varepsilon^{k-1} Y_{k}^{\prime}\left(\tau, y_{0}(\tau, \varepsilon)\right) .
$$

В дальнейшем нам потребуется

Лемма. Пусть система (10) при достаточно малых ненулевых $\varepsilon$ не имеет характеристических показателей с нулевой вещественной частью. Тогда дифференциальный оператор

$$
K(\varepsilon) y=\frac{d y}{d \tau}-A(\tau, \varepsilon) y
$$

имеет при достаточно малых $\varepsilon$ обратный оператор $K^{-1}(\varepsilon)$, определенный на пространстве почти периодических функиий, и

$$
\left\|K^{-1}(\varepsilon)\right\| \leqslant \frac{M}{\varepsilon^{\alpha}}, \quad M, \alpha>0 .
$$

ДокАЗАтЕЛЬСтво. Рассмотрим уравнение

$$
\frac{d y}{d \tau}=A(\tau, \varepsilon) y+f(\tau)
$$

где $f(\tau)$ - произвольная почти периодическая функция. Матрица $A(\tau, \varepsilon)-\omega$-периодическая по $\tau$; следовательно (см. [4, гл. 4]), существует линейная замена $y=P(\tau, \varepsilon) z$, приводящая (12) к виду

$$
\frac{d z}{d \tau}=R(\varepsilon) z+P^{-1}(\tau, \varepsilon) f(\tau)
$$


где $R(\varepsilon)$ - не зависящая от $\tau$ матрица, не имеющая при мальх $\varepsilon \neq 0$ собственных значений с нулевой вещественной частью. При этом матрица $R(0)$ имеет собственные значения с нулевой вещественной частью. Матрица $P(\tau, \varepsilon)$ - вещественная, невырожденная, достаточно гладкая по $\varepsilon$, периодическая по $\tau$ с периодом $T$ ( $T$ - целое, кратное $\omega)$ и $\|P(\tau, \varepsilon)\|,\left\|P^{-1}(\tau, \varepsilon)\right\| \leqslant C, C=$ const. Как известно, при этих условиях оператор

$$
L(\varepsilon) z=\frac{d z}{d \tau}-R(\varepsilon) z
$$

обратим в пространстве почти периодических функций при достаточно малых $\varepsilon$ и

$$
\left\|L^{-1}(\varepsilon)\right\| \leqslant \frac{M_{1}}{\varepsilon^{\alpha}}, \quad M_{1}, \alpha>0
$$

(см. [5]). Следовательно, уравнение (12) имеет при достаточно малых $\varepsilon$ единственное почти периодическое решение

$$
y^{*}(\tau, \varepsilon)=P(\tau, \varepsilon) L^{-1}(\varepsilon) P^{-1}(\tau, \varepsilon) f(\tau)
$$

причем

$$
\left\|y^{*}(\tau, \varepsilon)\right\| \leqslant \frac{C^{2} M_{1}}{\varepsilon^{\alpha}}\|f(\tau)\|,
$$

т.е. существует оценка вида (11). Лемма доказана.

Систему (5) запишем в виде

$$
\frac{d y}{d \tau}=A(\tau, \varepsilon) y+(H(\tau, y, \varepsilon)-A(\tau, \varepsilon) y)+\varepsilon^{m} F\left(\frac{\tau}{\varepsilon}, \tau, y, \varepsilon\right)
$$

где

$$
H(\tau, y, \varepsilon)=\sum_{k=1}^{m} \varepsilon^{k-1} Y_{k}(\tau, y), \quad A(\tau, \varepsilon)=H_{y}^{\prime}\left(\tau, y_{0}(\tau, \varepsilon), \varepsilon\right) .
$$

TEOPEMA. Пycmb

1) при $0<\varepsilon<\varepsilon_{1}$ все характеристические показатели системы (10) имеют ненулевые вещественные части и выполняется неравенство (11) с некоторыми константами $M, \alpha>0$;

2) при $0<\varepsilon<\varepsilon_{1}$ для некоторого иара $U\left(y_{0}(\varepsilon t, \varepsilon), r(\varepsilon)\right)$ пространства $B\left(\mathbb{R}^{n}\right)$ при $y_{1}(t), y_{2}(t) \in U\left(y_{0}(\varepsilon t, \varepsilon), r(\varepsilon)\right)$ выполняются неравенства

$$
\left\|H\left(\varepsilon t, y_{1}(t), \varepsilon\right)-H\left(\varepsilon t, y_{2}(t), \varepsilon\right)-A(\varepsilon t, \varepsilon)\left(y_{1}(t)-y_{2}(t)\right)\right\| \leqslant r(\varepsilon)\left\|y_{1}(t)-y_{2}(t)\right\|
$$

əде $r(\varepsilon)=c_{0} \varepsilon^{\eta}, c_{0}=$ const, $\eta>\alpha$,

$$
\left\|F\left(t, \varepsilon t, y_{1}(t), \varepsilon\right)-F\left(t, \varepsilon t, y_{2}(t), \varepsilon\right)\right\| \leqslant K_{0}\left\|y_{1}(t)-y_{2}(t)\right\|, \quad K_{0}=\mathrm{const}
$$

3) $m>\alpha+\eta$. 
Тогда при достаточно малых $\varepsilon$ система (1) имеет в шаре $U\left(y_{0}(\varepsilon t, \varepsilon), r(\varepsilon)\right)$ единственное почти периодическое решение $x_{0}(t, \varepsilon)$. Это решение будет асимптотически устойчиво, если при достаточно малых в все характеристические показатели системы (10) имеют отрицательные вещественные части, и неустойчиво в противном случае.

Прежде чем перейти к доказательству, остановимся подробнее на условиях теоремы. Предположим, что система (3) имеет мультипликаторы на единичной окружности. В этом случае изучения усредненной системы первого приближения (2) недостаточно, чтобы судить о существовании п.п.-решения системы (1) близкого к $y_{0}(\varepsilon t)$. С помощью процедуры, описанной в п. 2, будем строить системы более высоких приближений до тех пор, пока при некотором $m_{0}>1$ не будут выполнены условия теоремы. Требуется, чтобы при достаточно малых $\varepsilon>0$ все мультипликаторы соответствуюшей системы в вариациях (10) не лежали на единичной окружности. После этого необходимо найти константу $\alpha$, с которой вьполняется неравенство (11). Отметим, что $\alpha$ может оказаться нецелым. Число $\eta$ - порядок по $\varepsilon$ величины, характеризующей радиус шара, в котором находится искомое п.п.-решение, может быть выбрано произвольным, но с условием $\eta>\alpha$. Далее следует продолжить построение систем высших приближений для системы (1) до порядка $m \geqslant m_{0}$, для которого вьполняется неравенство $m>\alpha+\eta$.

ДокАЗАТЕльСтво теоремЫ. Так как замена (4) обратима при малых $\varepsilon$, достаточно доказать утверждения теоремы для системы (13). Задача о сушествовании п.п.-решений для системы (13) эквивалентна при достаточно малых $\varepsilon>0$ задаче о п.п.-решениях операторного уравнения

$$
y=\Pi(y, \varepsilon), \quad \Pi(y, \varepsilon)=K^{-1}(\varepsilon)(H(\tau, y, \varepsilon)-A(\tau, \varepsilon) y)+\varepsilon^{m} K^{-1}(\varepsilon) F\left(\frac{\tau}{\varepsilon}, \tau, y, \varepsilon\right) .
$$

Пусть $y_{0}(\tau, \varepsilon)$ - п.п.-решение системы $m$-го приближения (9), т.е.

$$
\frac{d y_{0}(\tau, \varepsilon)}{d \tau} \equiv H\left(\tau, y_{0}(\tau, \varepsilon), \varepsilon\right) .
$$

Рассмотрим функцию П $\left(y_{0}+z, \varepsilon\right)$, где $z$ - произвольная п.п.-функция такая, что $\|z\| \leqslant r(\varepsilon)$. Имеем

$$
\begin{aligned}
\Pi\left(y_{0}+z, \varepsilon\right)= & K^{-1}(\varepsilon)\left(H\left(\tau, y_{0}+z, \varepsilon\right)-A(\tau, \varepsilon)\left(y_{0}+z\right)\right)+\varepsilon^{m} K^{-1}(\varepsilon) F\left(\frac{\tau}{\varepsilon}, \tau, y_{0}+z, \varepsilon\right) \\
= & K^{-1}(\varepsilon)\left(H\left(\tau, y_{0}+z, \varepsilon\right)-H\left(\tau, y_{0}, \varepsilon\right)-A(\tau, \varepsilon) z\right) \\
& +\varepsilon^{m} K^{-1}(\varepsilon) F\left(\frac{\tau}{\varepsilon}, \tau, y_{0}+z, \varepsilon\right)+K^{-1}(\varepsilon)\left(H\left(\tau, y_{0}, \varepsilon\right)-A(\tau, \varepsilon) y_{0}\right) .
\end{aligned}
$$

Из определения оператора $K^{-1}(\varepsilon)$ следует, что

$$
K^{-1}(\varepsilon)\left(H\left(\tau, y_{0}, \varepsilon\right)-A(\tau, \varepsilon) y_{0}\right)=y_{0} .
$$

Следовательно,

$$
\begin{aligned}
\Pi\left(y_{0}+z, \varepsilon\right)= & K^{-1}(\varepsilon)\left(H\left(\tau, y_{0}+z, \varepsilon\right)-H\left(\tau, y_{0}, \varepsilon\right)-A(\tau, \varepsilon) z\right) \\
& +\varepsilon^{m} K^{-1}(\varepsilon) F\left(\frac{\tau}{\varepsilon}, \tau, y_{0}+z, \varepsilon\right)+y_{0} .
\end{aligned}
$$


Оценим норму п.п.-функции П $\left(y_{0}+z, \varepsilon\right)-y_{0}$. Получим

$$
\begin{gathered}
\left\|\Pi\left(y_{0}+z, \varepsilon\right)-y_{0}\right\| \leqslant\left\|K^{-1}(\varepsilon)\left(H\left(\tau, y_{0}+z, \varepsilon\right)-H\left(\tau, y_{0}, \varepsilon\right)-A(\tau, \varepsilon) z\right)\right\| \\
+\left\|\varepsilon^{m} K^{-1}(\varepsilon) F\left(\frac{\tau}{\varepsilon}, \tau, y_{0}+z, \varepsilon\right)\right\| .
\end{gathered}
$$

Из условий теоремы следует, что при $\varepsilon \rightarrow 0$

$$
\left\|\Pi\left(y_{0}+z, \varepsilon\right)-y_{0}\right\| \leqslant \Delta_{1}(\varepsilon)+\Delta_{2}(\varepsilon)
$$

где $\Delta_{1}(\varepsilon)=O\left(\varepsilon^{2 \eta-\alpha}\right), \Delta_{2}(\varepsilon)=O\left(\varepsilon^{m-\alpha}\right), 2 \eta-\alpha>\eta, m-\alpha>\eta$. Так как $r(\varepsilon)=c_{0} \varepsilon^{\eta}$, при достаточно малых $\varepsilon>0$ из $\|z\| \leqslant r(\varepsilon)$ следует, что $\left\|\Pi\left(y_{0}+z, \varepsilon\right)-y_{0}\right\| \leqslant r(\varepsilon)$. Это означает, что при достаточно малых $\varepsilon>0$ оператор $\Pi(y, \varepsilon)$ отображает шар $U\left(y_{0}, r(\varepsilon)\right)$ в себя. Покажем, что оператор П $(y, \varepsilon)$ при мальх $\varepsilon$ является сжимающим. Пусть $y_{1}, y_{2} \in$ $U\left(y_{0}, r(\varepsilon)\right)$. Оценим норму п.п.-функции $\Pi\left(y_{1}, \varepsilon\right)-\Pi\left(y_{2}, \varepsilon\right)$ :

$$
\begin{aligned}
\left\|\Pi\left(y_{1}, \varepsilon\right)-\Pi\left(y_{2}, \varepsilon\right)\right\| \leqslant & \left\|K^{-1}(\varepsilon)\right\| \cdot\left\|H\left(\tau, y_{1}, \varepsilon\right)-H\left(\tau, y_{2}, \varepsilon\right)-A(\tau, \varepsilon)\left(y_{1}-y_{2}\right)\right\| \\
& +\varepsilon^{m}\left\|K^{-1}(\varepsilon)\right\| \cdot\left\|F\left(\frac{\tau}{\varepsilon}, \tau, y_{1}, \varepsilon\right)-F\left(\frac{\tau}{\varepsilon}, \tau, y_{2}, \varepsilon\right)\right\| .
\end{aligned}
$$

Из условий теоремы следует, что при $\varepsilon \rightarrow 0$

$$
\left\|\Pi\left(y_{1}, \varepsilon\right)-\Pi\left(y_{2}, \varepsilon\right)\right\| \leqslant\left(\Delta_{3}(\varepsilon)+\Delta_{4}(\varepsilon)\right)\left\|y_{1}-y_{2}\right\|,
$$

где $\Delta_{3}(\varepsilon)=O\left(\varepsilon^{\eta-\alpha}\right), \Delta_{4}(\varepsilon)=O\left(\varepsilon^{m-\alpha}\right), \eta-\alpha>0, m-\alpha>0$. Следовательно, при достаточно малых $\varepsilon>0$ оператор $\Pi(y, \varepsilon)$ - сжимающий на шаре $U\left(y_{0}, r(\varepsilon)\right)$. Таким образом, уравнение (14) имеет при достаточно мальх $\varepsilon$ в шаре $U\left(y_{0}, r(\varepsilon)\right)$ единственное п.п.-решение. Существование п.п.-решения $x(t, \varepsilon)$ для уравнения $(1)$ доказано. Доказательство утверждений об устойчивости решения $x(t, \varepsilon)$ аналогично доказательству теорем об устойчивости и неустойчивости по первому приближению (см., например, $[6, \S 11])$.

\section{СПИСОК ЦИТИРОВАННОЙ ЛИТЕРАТУРЫ}

[1] Roseau M. Sur une classe des systemes dynamiques soumis à des excitations periodiques de longue periode // C. R. Acad. Sci. Paris. Sér. A. 1969. V. 268. № 7. P. 409-412.

[2] Бурд В.Ш. Малые почти периодические колебания в системах с быстрыми и медленными параметрическими возбуждениями // Деп. ВИНИТИ. М.: ВИНИТИ, 1982. № 176-83.

[3] Левитан Б. М. Почти-периодические функции. М.: Гостехиздат, 1953.

[4] Якубович В. А., Старжинский В. М. Линейные дифференциальные уравнения с периодическими коэффициентами и их приложения. М.: Наука, 1972.

[5] Штокало И.З. Линейные дифференциальные уравнения с переменными коэффициентами. Киев: Изд-во АН УССР, 1960.

[6] Красносельский М. А., Бурд В.Ш., Колесов Ю. С. Нелинейные почти периодические колебания. М.: Наука, 1970. 